\title{
Experiment and Finite Analysis on Resonant Bending Fatigue of Marine Risers
}

\author{
Fang Xiaoming, Yan Zhichao*, Wang Liquan and Huang Yuxuan \\ College of Mechanical and Electrical Engineering, Harbin Engineering University, Harbin, Heilongjiang, 150001, P.R. \\ China
}

\begin{abstract}
Riser system is a key equipment for offshore oil and gas development. When conducting riser design, fatigue failure mode is the chief one among the many failure modes which should be taken into account. To assess the fatigue performance of riser accurately, it is necessary to conduct fatigue tests. Resonant bending fatigue test is one effective method for fatigue tests of risers. In this paper, the principle of resonant bending fatigue test and test procedures are presented firstly, and then a finite element model using ABAQUS is created to simulate the resonant bending fatigue test, and the results from the finite element model are compared with the experimental results. The good agreements between the FEM results and experimental results verify the accuracy of the finite element model in this paper.
\end{abstract}

Keywords: Dynamic response, finite element analysis component. risers, resonant bending fatigue test.

\section{INTRODUCTION}

Riser system is a special steel pipe string connected offshore platform and subsea equipments located in the seabed. Riser system is a key component of offshore oil and gas development and is one of the most complex equipments in subsea production. Riser system is very fragile in deepwater environment, which includes five typical failure modes: fatigue failure, corrosion failure, erosion failure, pipe choking/flow limit failure and pipe joint failure [1]. In the above five kinds of failure modes, the first consideration is the fatigue failure [2]. Therefore, in the course of the development of the riser system, fatigue tests must be conducted to verify the expected fatigue life, and determine the key area of riser fatigue to make necessary improvements [3].

Aimed at the dynamic characteristics and dynamic responses of marine risers, international and domestic scholars have conducted a lot of research and have accumulated valuable experience in theory and engineering applications. Professor Bai Yong, etc. summarized their research contents in the aspect of offshore pipe and riser system, which includes riser fatigue caused by VIV (vortexinduced vibrations) [1]. Professor Duan Menglan, etc. conducted in-depth research in the fatigue fracture and reliability assessment of deepwater risers [4]. Professor Guo Haiyan, etc. did a lot of research in terms of the subsea riser VIV of internal flow [5-7]. Professor Chen Guoming, etc. reviewed the results of the foreign and domestic research concerning subsea riser fatigue tests [8]. Jeroen, etc. proposed a simplified mathematical model aimed at the resonant bending fatigue tests of pipes and did experimental research [9]. L. Bertini, etc. conducted experimental research of the bending fatigue aimed at marine riser joints [10].

As mentioned above, many scholars have done in-depth studies on the theoretical and experimental aspects of the marine riser resonance and fatigue failure, but the content does not involve simulation of the dynamic responses of a complete set of subsea riser bending fatigue test devices. Therefore, the author firstly introduces the principles of the riser bending fatigue tests and test procedures in this paper, then the FEM methods of the resonance subsea riser bending fatigue tests by the use of ABAQUS is introduced. This paper has carried on comparative analysis between the numerical simulation results and the experimental results, which shows good agreement between the two and thus to verify the accuracy of the finite element model used to simulate this type of riser fatigue tests in this paper.

\section{EASE OF USE RESONANT BENDING FATIGUE TEST}

The methods of the marine riser fatigue test can be divided into axial tensile fatigue test and bending fatigue test. The axial tensile fatigue test is mainly used for material fatigue test of risers, while the bending fatigue test is mainly used for structure fatigue test of risers, which mainly includes four-point bending test, rotating bending test and resonant bending test. The advantage of the resonant bending test is the high experimental frequency, which can reduce the time required to a large extent. Therefore, it is the mainstream approach at present of the riser full-scale fatigue tests [8]. This paper adopts the resonant bending test to measure fatigue performance of risers.

\subsection{Test Principles}

The principle of the resonant bending fatigue test is: exert rotating excitation load perpendicular to the pipe axis plane 


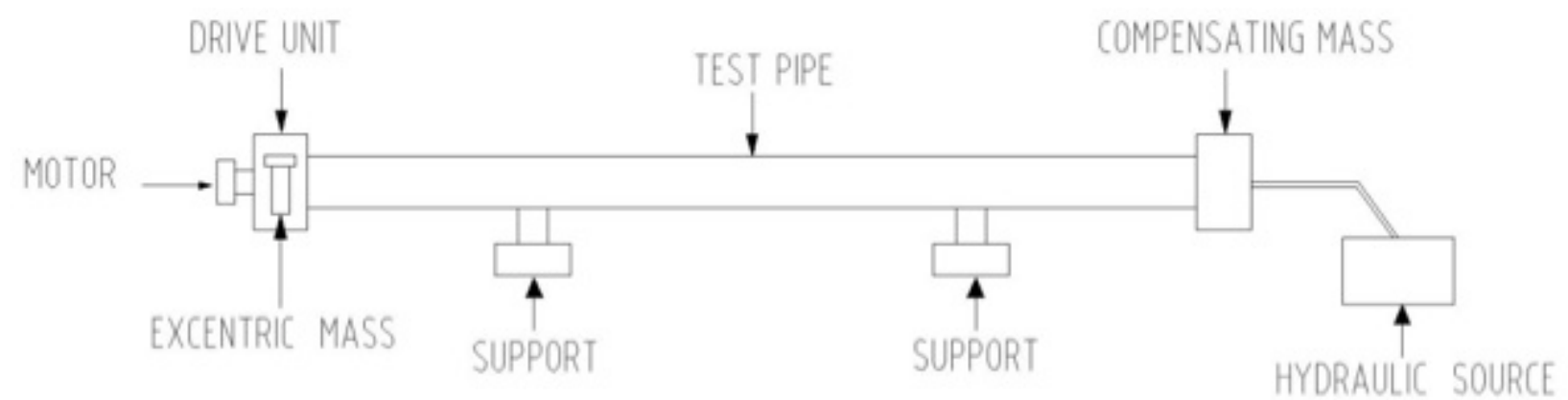

Fig. (1). Testing machine model of resonant bending fatigue.

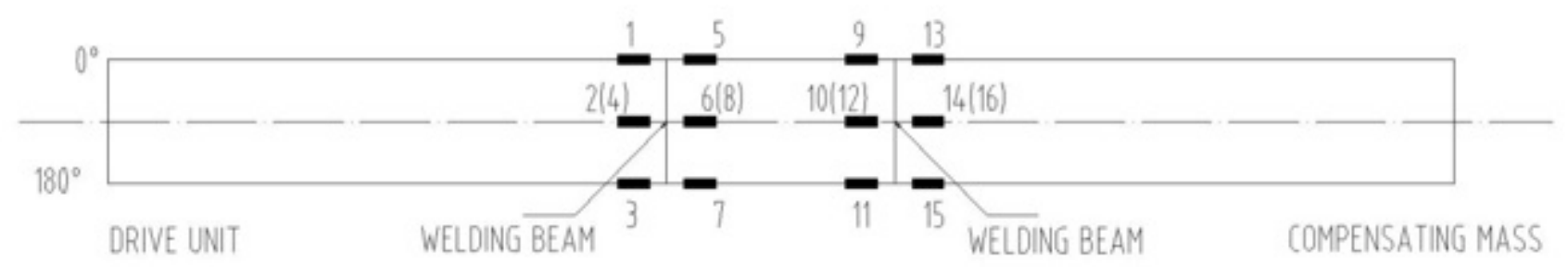

Fig. (2). Layout of the strain gauges.

on one end of the test sample pipe. The frequency of the excitation loads is close to the first natural frequency of the test sample pipe, which brings about resonance of the test sample pipe. In the process of vibration, there are alternating stresses on the cross-section of the pipe, which results in a fatigue damage.

\subsection{Test Devices}

The testing machine model of the resonant bending fatigue is as shown in Fig. (1). The major components of the testing machine include a test sample pipe, two supports, a eccentric mass, a balance mass, a motor and a hydraulic pump. Before the test, make sure the suitable length and natural frequency (from $20 \mathrm{~Hz}$ to $30 \mathrm{~Hz}$ in general) of the sample pipe as well as the location of the pipe's support point by the FEM calculation. In addition, paste strain gauges on the place which is near the weld of the pipe. There are four groups of strain gauges that amounts to 16 which is located at $0^{\circ}, 90^{\circ}, 180^{\circ}$ and $270^{\circ}$ of the pipe body. The layout of the strain gauges is as shown in Fig. (2). Before pasting the strain gauges, it is necessary to measure the sizes of the wall thickness, the diameter and so on of which are pasted strain gauges. These values are used to calculate stress concentration factor (SCF) caused by the pipe's initial defects. The calculation formula is as shown in (1) and the value will be used in the FEM calculation to solve the real stress of the pipe.

$S C F=1+2.6 \frac{e}{T_{1}}\left[\frac{1}{1+0.7\left(T_{2} / T_{1}\right)^{1.4}}\right]$

$e$ : Pipe shaft eccentricity value

$T_{1}$ : Minimum wall thickness

$T_{2}$ : Maximum wall thickness

During the test, inject internal pressure into the sample pipe to achieve specified values through the hydraulic pump, and adjust the motor speed to make the driving frequency close to the natural frequency of the pipe (approximately 0.95 times). Record the related data by the software. When the value of the hydraulic pressure appears a sudden drop or there occurs a leakage on the body of the pipe, the sample pipe can be seemed to have been destroyed. The physical map of this test is as shown in Fig. (3).

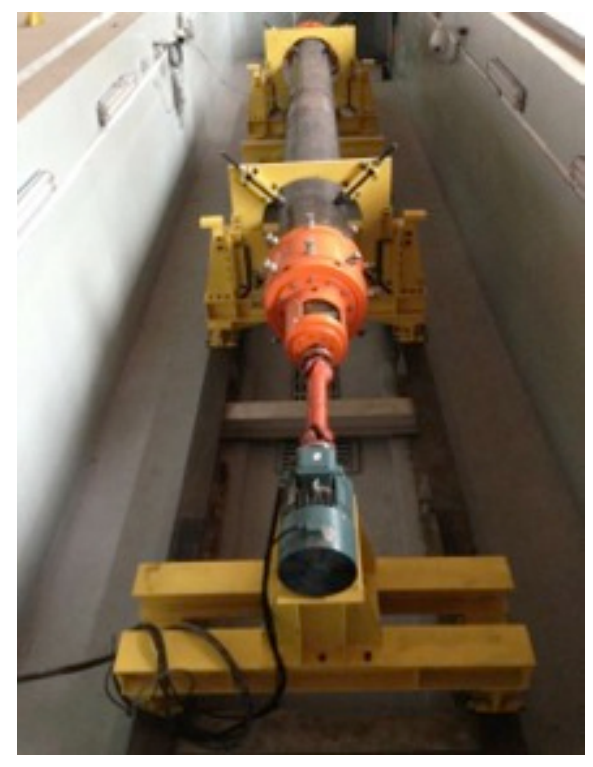

Fig. (3). Resonant bending test devices.

\subsection{Test Procedures}

The main procedures of this test include:

(1) Measure and record the sizes of the sample pipe, which include the pipe's outer diameter and wall thickness where are pasted strain gauges.

(2) Paste strain gauges on the specified location. 
(3) Install the sample pipe, and exert internal pressure on the sample pipe to the specified value.

(4) Zero the reading of the strain gauges.

(5) Adjust the revolving speed of the driving motor to the specified value.

(6) Entry data including the value of the strain gauges, the pipe's internal pressure, test frequency, cycleindex and so on.

(7) Cycle loading until the sample pipe is destroyed (there occurs a leakage), and record the strain amplitude as well as the total times of cycles.

(8) Mark the locations destroyed and dispose of the data recorded.

\section{THEORETICAL MODEL AND FINITE ELEMENT ANALYSIS}

\subsection{Theoretical Model}

The model of this research is finite length cylindrical shells, whose geometry and coordinate system are shown in Fig. (4). Cylindrical shell casing surface radius is $\mathrm{R}$, thickness is $h$, shell length is $L$. Using $(x, \theta)$ of the cylindrical coordinate system, u, v, w are the cylindrical shell in the axial, circumferential, radial displacement.

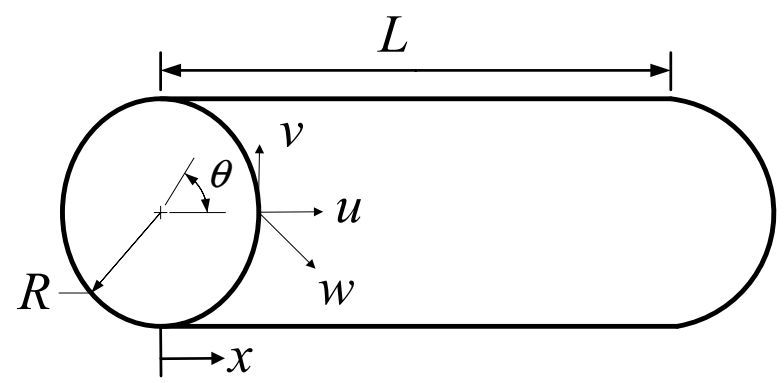

Fig. (4). Cylindrical shell geometry \& Coordinate system model.

$$
\begin{aligned}
& \frac{\partial^{2} u}{\partial x^{2}}+\frac{(1-\mu)}{2 R^{2}}\left(1+\beta^{2}\right) \frac{\partial^{2} u}{\partial \theta^{2}}+\frac{(1+\mu)}{2 R} \frac{\partial^{2} v}{\partial x \partial \theta}+\frac{\mu}{R} \frac{\partial w}{\partial x} \\
& -\beta^{2} R \frac{\partial^{3} w}{\partial x^{3}}+\beta^{2} \frac{(1-\mu)}{2 R} \frac{\partial^{3} w}{\partial x \partial \theta^{2}}-\frac{1}{c_{L}^{2}} \frac{\partial^{2} u}{\partial t^{2}}=0 \\
& \frac{(1+\mu)}{2 R} \frac{\partial^{2} u}{\partial x \partial \theta}+\frac{(1-\mu)}{2} \frac{\partial^{2} v}{\partial x^{2}}+\frac{1}{R^{2}} \frac{\partial^{2} v}{\partial \theta^{2}}+\frac{1}{R^{2}} \frac{\partial w}{\partial \theta}+ \\
& \beta^{2}\left(\frac{3(1-\mu)}{2} \frac{\partial^{2} v}{\partial x^{2}}-\frac{(3-\mu)}{2} \frac{\partial^{3} w}{\partial x^{2} \partial \theta}\right)-\frac{1}{c_{L}^{2}} \frac{\partial^{2} v}{\partial t^{2}}=0 \\
& \beta^{2}\left(\begin{array}{l}
R^{2} \frac{\partial^{4} w}{\partial x^{4}}+2 \frac{\partial^{4} w}{\partial x^{2} \partial \theta^{2}}+\frac{1}{R^{2}} \frac{\partial^{4} w}{\partial \theta^{4}}-R \frac{\partial^{3} u}{\partial x^{3}}+ \\
\frac{(1-\mu)}{2 R} \frac{\partial^{3} u}{\partial x \partial \theta^{2}}-\frac{(3-\mu)}{2} \frac{\partial^{3} v}{\partial x^{2} \partial \theta}+\frac{2}{R^{2}} \frac{\partial^{2} w}{\partial \theta^{2}}
\end{array}\right) \\
& +\frac{\mu}{R} \frac{\partial u}{\partial x}+\frac{1}{R^{2}}\left(\frac{\partial v}{\partial \theta}+w\left(1+\beta^{2}\right)\right)+\frac{1}{c_{L}^{2}} \frac{\partial^{2} w}{\partial t^{2}}=0
\end{aligned}
$$

$$
\begin{aligned}
& \text { Where Aij (i, } j=1,2,3) \text { can expressed as below: } \\
& A_{11}=\Omega^{2}+\left(k_{n} R\right)^{2}-\frac{(1-\mu)}{2} n^{2}\left(1+\beta^{2}\right) \\
& A_{12}=\frac{(1+\mu)}{2} n\left(k_{n} R\right) \\
& A_{13}=\mu\left(k_{n} R\right)-\beta^{2}\left(k_{n} R\right)^{3}-\frac{(1-\mu)}{2} \beta^{2} n^{2}\left(k_{n} R\right) \\
& A_{21}=-A_{12} \\
& A_{22}=\Omega^{2}+\frac{(1-\mu)}{2}\left(k_{n} R\right)^{2}\left(1+3 \beta^{2}\right)-n^{2} \\
& A_{23}=-n+\frac{(3-\mu)}{2} \beta^{2} n\left(k_{n} R\right)^{2} \\
& A_{31}=A_{13} \\
& A_{32}=-A_{23}
\end{aligned}
$$

where $k$ is the axial wave number, $\omega$ is the circular ency; $n$ is the number of modes for the week; $u, v, w$, is week are $1,2,3$, their cicumfention seen in Fig. (5). Because the cylindrical shell is circumferential closed, circumferential exists is the since boundary conditions are closely related.
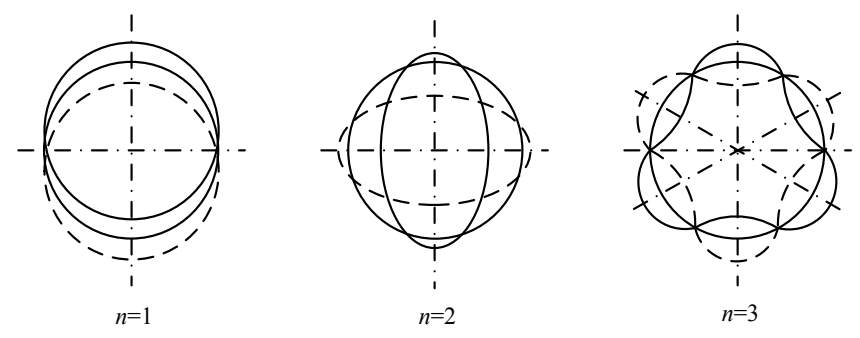

Fig. (5). Circumferential vibration mode of Cylindrical Shells.

$\left[\begin{array}{lll}A_{11} & A_{12} & A_{13} \\ A_{21} & A_{22} & A_{23} \\ A_{31} & A_{32} & A_{33}\end{array}\right]\left\{\begin{array}{c}U \\ V \\ W\end{array}\right\}=\{0\}$ 
$A_{33}=-\Omega^{2}+1+\beta^{2}\left\{\left[\left(k_{n} R\right)^{2}-n^{2}\right]^{2}+\left(1-2 n^{2}\right)\right\}$

where $\Omega=\omega R / c_{L}{ }^{2}=\omega R \sqrt{\left(1-\mu^{2}\right) \rho / E}$ is dimensionless frequency parameter.

The condition of formula (8) have nonzero solution is that the coefficient matrix determinant is 0 .

$\operatorname{det}\left(\left[A_{i j}\right]\right)=\left|\begin{array}{ccc}A_{11} & A_{12} & A_{13} \\ A_{21} & A_{22} & A_{23} \\ A_{31} & A_{32} & A_{33}\end{array}\right|=0$

Through finishing on (9), we can get an equation on axial wave number and the dimensionless frequency:

$f\left(k_{n} R, \Omega\right)=0$

\subsection{Finite Element Analysis}

In this paper, the finite element is modeled by the general FEM software--ABAQUS. In order to reduce the computation load, the pipe sample takes the beam element (pipe31) model here, and the weights of the ends and central connection flanges of which are equivalent to the material points pressed on it. The connection between the pipe sample and the supports is defined as a linear spring.

\subsubsection{Methods of Analysis}

There are totally two kinds of analysises for this pipe modle.

(1) To solve the natural frequency and the natural vibration mode of the structure, select the appropriate length of the pipe sample to control the natural frequencies within the range of $20 \mathrm{~Hz}-30 \mathrm{~Hz}$, and the support locations of pipe sample needs to be determined.
On the basis of the natural frequency and vibration mode, choose the excitation load close to the natural frequency of the structure to act on one end of pipe, and solve the vibration response of the structure.

\subsubsection{Effect of Load}

In this pipe model, totally four kinds of loads in the following are to be considered:

(1) Gravity load on the whole model.

(2) Water pressure load on the inner surface of pipe.

(3) Axial tension load on the pipe ends from the inner water pressure of pipe.

(4) Rotation excitation force load on one end of the pipe sample, which can be shown in formula (11). In this model, the rotation excitation force can be decomposed into two mutually perpendicular component forces, and changing in accordance with the laws of sine and cosine respectively.

$F_{e}(t)=m_{e} \cdot r_{e} \cdot \omega_{e}{ }^{2} \cdot e^{i \cdot \omega_{e} t}$

$m_{e}$ : Eccentric block mass

$r e:$ Eccentric distance

$\omega_{e}$ : Angular velocity of rotation excitation

\section{RESULTS ANALYSIS}

\subsection{The Experimental Data and Results Analysis}

During the test, the pipe body strain measurements and cycles are input by software automatically. After vibration is steady, the difference between the average maximum and the average minimum recorded can be the strain amplitude, as shown in Table 1. Strain amplitude value multiplied by elastic modulus $(210000 \mathrm{MPa})$ is the stress amplitude, as shown in Table 2.

Table 1. SG1-SG16 strain amplitude (orientation survey).

\begin{tabular}{|l|c|c|c|c|c|c|}
\hline Strain Gauges No. & SG1 & SG2 & SG3 & SG4 & SG5 & SG6 \\
\hline Strain Amplitude (10E-6) & 548 & 537 & 552 & 535 & 617 & 628 \\
\hline Strain Gauges No. & SG7 & SG8 & SG9 & SG10 & SG11 & SG12 \\
\hline strain Amplitude (10E-6) & 626 & 637 & 573 & 596 & 567 & 584 \\
\hline Strain Gauges No. & SG13 & SG14 & SG15 & SG16 & & \\
\hline strain Amplitude (10E-6) & 480 & 490 & 478 & 497 & & \\
\hline
\end{tabular}

Table 2. SG1-SG16 stress amplitude (orientation survey).

\begin{tabular}{|l|c|c|c|c|c|c|}
\hline Strain gauges No. & SG1 & SG2 & SG3 & SG4 & SG5 & SG6 \\
\hline Stress amplitude (MPa) & 115 & 113 & 116 & 112 & 130 & 132 \\
\hline Strain gauges No. & SG7 & SG8 & SG9 & SG10 & SG11 & SG12 \\
\hline Stress amplitude (MPa) & 131 & 134 & 120 & 125 & 119 & 123 \\
\hline Strain gauges No. & SG13 & SG14 & SG15 & SG16 & & \\
\hline Stress amplitude (MPa) & 101 & 103 & 100 & 104 & \\
\hline
\end{tabular}


After several cycles, the test sample pipe leak near the weld, as shown in Fig. (6), that's called the destruction of the sample. After recording the cycle times number is 854,588 .
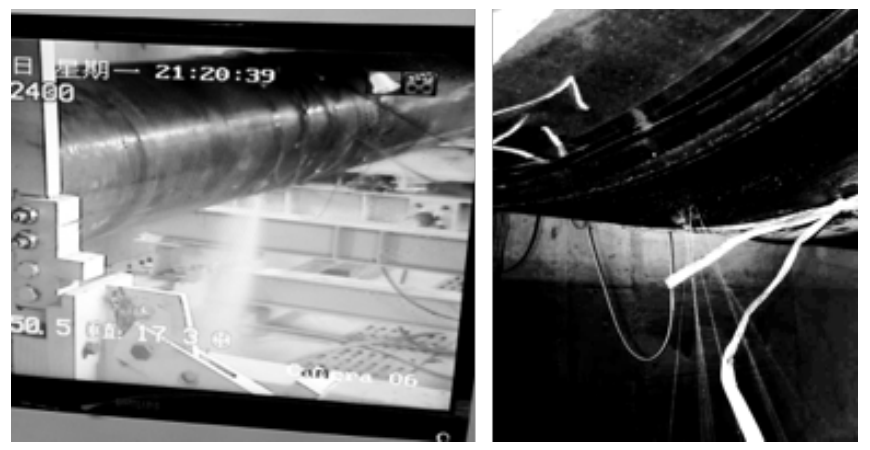

Fig. (6). Sample leak condition.

The pipe body destroy at the location close to the strain gauge 5-8's circumference. Therefore the strain amplitude average value of strain gauge 5-8 is the strain amplitude of the damage position. Comparing the results with s-n curve in BS7608[11] and DNV RP C203[12] specifications, as shown in Fig. (7). Results showed that the test cycles numerical value is between DNV RP C203 d-s-n curve and the DNV RP C203 C2-s-n curve.

\subsection{Finite Element Analysis Of Simulation Results}

Calculated based on ABAQUS, we can get a natural frequency of the structure, which is $22.77 \mathrm{~Hz}$.

Infinite element model, the corresponding element and experimental strain gage variation of stress amplitude is shown in Table 3, data recording can be seen in Table 4.

Considering the influence of initial defects of the pipe, the nominal stress multiplied by the stress concentration factor (SCF), which can be the real stress of a unit. SCF formula can be seen in (1), which is mentioned before, the measurement data and the calculated values are shown in Table $\mathbf{5}$ and real stress amplitude are shown in Table $\mathbf{6}$.

\section{COMPARISON OF THE RESULTS}

The comparison between test results and the results of finite element can be seen in Table 7.

As shown in the previous table, in finite element model, the mean average stress amplitude at damaged areas is 138 $\mathrm{MPa}$, and compared with the experimental results (132 $\mathrm{MPa}$ ), the relative error is $4.5 \%$. Among all 16 comparisons, the relative errors are within $15 \%$. The cause of the error has the following aspects:

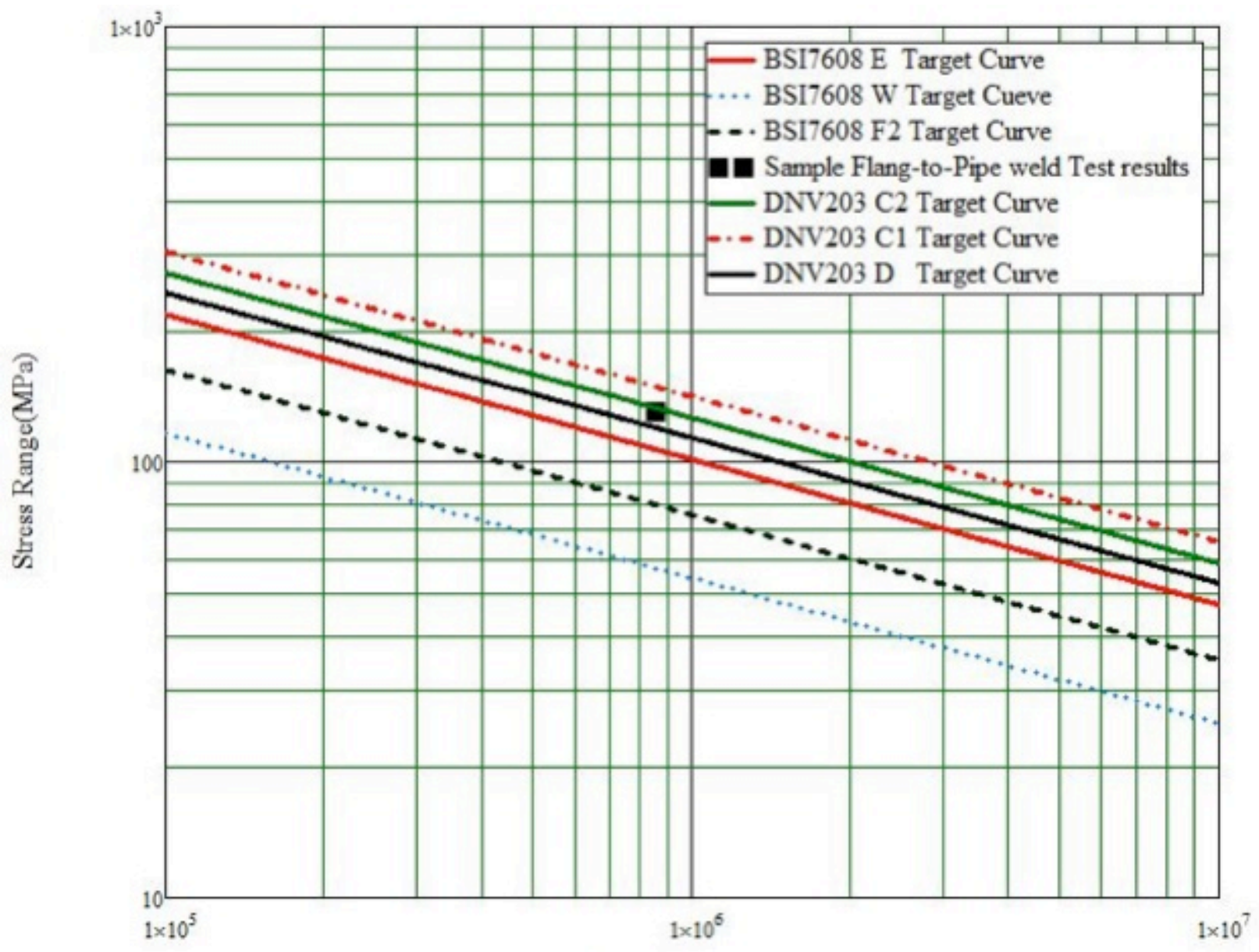

Number of cycles

Fig. (7). Comparison of fatigue test results. 
Table 3. SG1-SG16 variation of stress amplitude (finite element model).

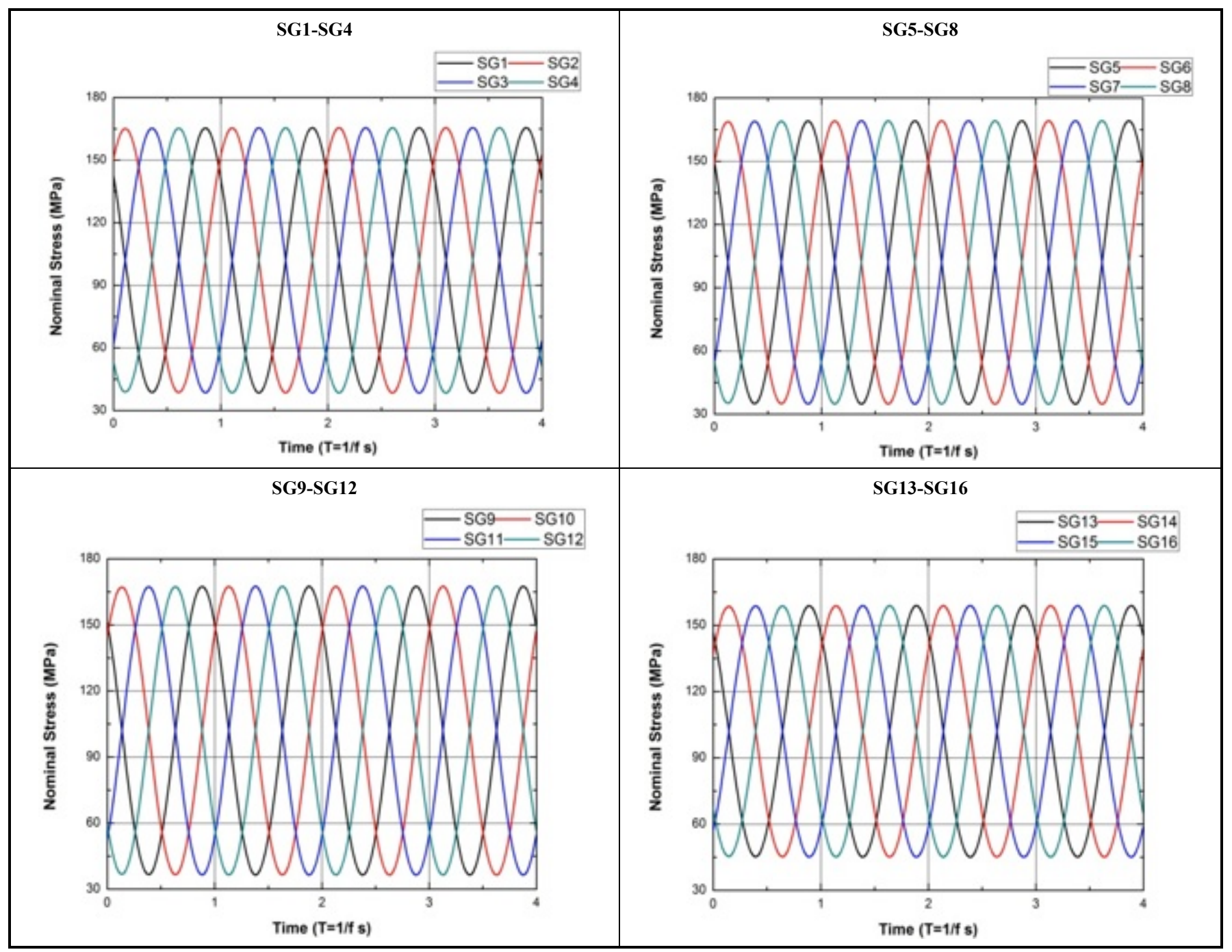

Table 4. SG1-SG16 normal stress amplitude (finite element model).

\begin{tabular}{|l|c|c|c|c|}
\hline Strain Gauges No. & SG1-SG4 & SG5-SG8 & SG9-SG12 & SG13-SG16 \\
\hline Nominal Stress Amplitude (MPa) & 127 & 134 & 131 & 114 \\
\hline
\end{tabular}

(1) Damping of the test environment cannot be accurately simulated in the finite element model.

(2) In finite element model, end weight equivalent for particles can cause errors.

\section{CONCLUSION}

At first, this paper introduces the experimental method and steps of pipe resonant bending fatigue test. Then it describes the use of finite element software ABAQUS to carry on finite element simulation of the dynamic response of the riser bending resonance method Finally the test results with the finite element results are compared, It can obtain the following conclusions:
(1) Through the resonant bending fatigue test pipe samples, the amplitude of stress in $132 \mathrm{MPa}$, after 854588 cycles failure. The number of cycles between cycles between DNV C203 D S-N curve and DNV C203 type C2 S-N curve, close to the DNVC203C2 type S-N curve, the concrete results as shown in Fig. (6).

(2) In the finite element model, the emergence of destruction the amplitude averaged $138 \mathrm{MPa}$, compared with the test results of the relative error was $4.5 \%$; 16 groups comparing the finite element results with the experimental results, the relative errors are within $15 \%$, and shows that finite element method in this paper has the reliability, can be used to analyze this kind of vertical resonance pipe fatigue the bending response test. 
Table 5. Stress concentration factor measurement data.

\begin{tabular}{|c|c|c|c|c|}
\hline $\begin{array}{c}\text { Strain Gauges } \\
\text { No. }\end{array}$ & $\begin{array}{c}\text { Minimum Wall } \\
\text { Thickness } \mathbf{T}_{\mathbf{1}} \text { (inch) }\end{array}$ & $\begin{array}{c}\text { Maximum Wall } \\
\text { Thickness } \mathbf{T}_{\mathbf{2}} \text { (inch) }\end{array}$ & $\begin{array}{c}\text { Eccentric Value } \\
\text { (inch) }\end{array}$ & $\begin{array}{c}\text { Stress Concentration } \\
\text { Factor SCF }\end{array}$ \\
\hline \hline SG1 & 0.972 & 0.974 & 0.006 & 1.01 \\
\hline SG2 & 0.972 & 0.981 & 0.0005 & 1.00 \\
\hline SG3 & 0.972 & 0.978 & 0.009 & 1.01 \\
\hline SG4 & 0.940 & 0.978 & 0.001 & 1.03 \\
\hline SG5 & 0.974 & 0.978 & 0.022 & 1.03 \\
\hline SG6 & 0.980 & 0.991 & 0.023 & 1.04 \\
\hline SG8 & 0.970 & 0.974 & 0.019 & 1.03 \\
\hline SG9 & 0.983 & 0.987 & 0.011 & 1.01 \\
\hline SG10 & 0.939 & 0.973 & 0.006 & 1.03 \\
\hline SG11 & 0.927 & 0.974 & 0.017 & 1.03 \\
\hline SG12 & 0.928 & 0.972 & 0.020 & 1.01 \\
\hline SG13 & 0.927 & 0.977 & 0.010 & 1.01 \\
\hline SG15 & 0.980 & 0.989 & 0.006 & 1.01 \\
\hline SG16 & 0.973 & 0.974 & 0.008 & 1.00 \\
\hline
\end{tabular}

Table 6. Real stress amplitude (finite element model).

\begin{tabular}{|l|c|c|c|c|c|c|}
\hline Strain Gauges No. & SG1 & SG2 & SG3 & SG4 & SG5 & SG6 \\
\hline Nominal Stress Amplitude (MPa) & 127 & 127 & 127 & 127 & 134 \\
\hline Real Stress Amplitude (MPa) & 128 & 127 & 128 & 127 & 138 \\
\hline Strain Gauges No. & SG7 & SG8 & SG9 & SG10 & SG11 & SG12 \\
\hline Nominal Stress Amplitude (MPa) & 134 & 134 & 131 & 131 & 131 \\
\hline Real Stress Amplitude (MPa) & 139 & 138 & 134 & 132 & 135 & 135 \\
\hline Strain Gauges No. & SG13 & SG14 & SG15 & SG16 & 114 & \\
\hline Nominal Stress Amplitude (MPa) & 114 & 114 & 114 & 114 & & \\
\hline Real Stress Amplitude (MPa) & 115 & 115 & & & \\
\hline
\end{tabular}

Table 7. The Test results Compared With The Results of Finite Element.

\begin{tabular}{|c|c|c|c|c|c|c|}
\hline Strain Gauges No. & SG1 & SG2 & SG3 & SG4 & SG5 & SG6 \\
\hline Test Stress Amplitude (MPa) & 115 & 113 & 116 & 112 & 130 & 132 \\
\hline Finite Element Stress Amplitude (MPa) & 128 & 127 & 128 & 127 & 138 & 138 \\
\hline Relative Error & $11.3 \%$ & $12.4 \%$ & $10.3 \%$ & $13.4 \%$ & $6.2 \%$ & $4.5 \%$ \\
\hline Strain Gauges No. & SG7 & SG8 & SG9 & SG10 & SG11 & SG12 \\
\hline Test Stress Amplitude (MPa) & 131 & 134 & 120 & 125 & 119 & 123 \\
\hline Finite Element Stress Amplitude (MPa) & 139 & 138 & 134 & 132 & 135 & 135 \\
\hline Relative Error & $6.1 \%$ & $3.0 \%$ & $11.7 \%$ & $5.6 \%$ & $13.4 \%$ & $9.8 \%$ \\
\hline Strain Gauges No. & SG13 & SG14 & SG15 & SG16 & & \\
\hline Test Stress Amplitude (MPa) & 101 & 103 & 100 & 104 & & \\
\hline Finite Element Stress Amplitude (MPa) & 115 & 115 & 115 & 114 & & \\
\hline Relative Error & $13.9 \%$ & $11.7 \%$ & $15 \%$ & $9.6 \%$ & & \\
\hline
\end{tabular}




\section{CONFLICT OF INTEREST}

The authors confirm that this article content has no conflict of interest.

\section{ACKNOWLEDGEMENTS}

Declared none.

\section{REFERENCES}

[1] Y. Bai, and Q. Bai, "Subsea Pipes and Risers", Elsevier Ocean Engineering Book Series, vol. 2, pp. 18-25, 2005.

[2] T. K. Sen, and K. Brown \& Root, "Fatigue in deep water steel catenary risers - A Probabilistic Approach for Assessment of Risk", In: Offshore Technology Conference, paper 19425, Houston, Texas, USA, 2008.

[3] API SPEC 16R, "Specification for Marine Drilling Riser Couplings," American Petroleum Institute, 1997.

[4] B. Xie, M. Duan, T. Qin, Z. Sun, and J. Li, "Advance of Research on Fatigue Fracture And Reliability Assessment of Deepwator Risers”, Acta Petrolei Sinica, vol. 25, no. 3, pp. 95-100, May 2004.
[5] H. Guo, and S. Wang, "Dynamic Characteristics of Marine Risers Conveying Fluid", China Ocean Engineering, vol. 14, no. 2, pp. 153-160, 2000.

[6] H. Guo, Y. Wang, and Q. Fu, "The Effect of Internal Fluid on The Response of Vortex-induced Vibration of Marine Riser", China Ocean Engineering, vol. 18, no. 1, pp 11-20, 2004.

[7] H. Guo, M. Lou, X. Dong, and X. Qi, "Numerical and Physical Investigation on Vortex-induced Vibration of Marine Risers", China Ocean Engineering, vol. 20, no. 3, 2006.

[8] X. Liu, G. Chen, Y. Chang, and S. Ju, "Fatigue Test Methods for Marine Risers", In: China Offshore Equipment Developing Forum, Tijin: China, pp. 71-75, 2011.

[9] J.V. Wittenberghe, P.D. Baets, W.D. Waele, W. Ost, M. Verstracte, and S. Hertele, "Resonant bending fatigue test setup for pipes with optical displacement measuring system," Journal of Offshore Mechanics and Arctic Engineering, pp. 013702-031706, vol. 134, no. 3, 2012.

[10] L. Bertini, M. Beghini, C. Santus, and A. Baryshnikov, "Resonant Test Rigs for Fatigue Full Scale Testing of Oil Drill String Connections". International Journal of Fatigue, vol. 30, no. 6, pp. 978-988, 2007.

[11] BS7608, "Codes of Practice for Fatigue Design and Assessment of Steel Structures," 1993.

[12] DNV RP C203, "Fatigue Design of Offshore Steel Structures," DET NORSKE VERITAS, 2010.

(C) Xiaoming et al.; Licensee Bentham Open.

This is an open access article licensed under the terms of the Creative Commons Attribution Non-Commercial License (http://creativecommons.org/licenses/by-nc/3.0/) which permits unrestricted, non-commercial use, distribution and reproduction in any medium, provided the work is properly cited 